\title{
Resection of Recurrent Carcinomas in the Pelvis - A New Field of Computer Aided Surgery
}

\author{
J. Geerling ${ }^{1}$, T. Hüfner ${ }^{1}$, R. Raab ${ }^{3}$, M. Citak ${ }^{1}$, T. Pohlemann ${ }^{4}$, H. Rosenthal ${ }^{2}$, \\ C. Krettek ${ }^{1}$ \\ ${ }^{1}$ Trauma Dept., Hannover Medical School, Carl-Neuberg-Str. 1, D-30625 Hannover \\ jens.geerling@planet-interkom.de \\ ${ }^{2}$ Radiology Dept., ${ }^{3}$ Dept. of Surgery, Hannover Medical School, D-30625 Hannover \\ ${ }^{4}$ Trauma Department, University of Saarland, 66421 Homburg, Germany
}

\begin{abstract}
Recurrent carcinomas may infiltrate the bony pelvis. If infiltrating the sacrum it is difficult to resect the tumor cause of the nerve roots that may be damaged during the operation.

In this study three cases of recurrent carcinomas infiltrating the sacrum are presented. All were resected using a navigation system (Surgigate, Medivision, Oberdorf, Switzerland). With this system it was possible to detect the tumor margins on the monitor screen and to perform a resection outside the margins of the tumor using navigated chissels.

Further developments such as CT and MRI fusion may help to improve such surgical procedures and may allow extend indications for computer aided surgery also in tumor cases.
\end{abstract}

\section{Objective}

The bony pelvis, especially the sacrum can be infiltrated by recurrent carcinomas of the rectum or the genitourinary system. The danger of a wide resection with an increased rate of nerval complications may be implyed by scar tissues from previous operations or radiation. Computer assisted surgery (CAS) could be a solution to increase the accuracy of tumor resections. Currently, navigation systems use the precise Computed tomography data allowing procedures like navigated pedicle screw applications, cup placement in hip arthroplasty and other procedures [1,2].

Navigated chisels or drilling machines may allow to extend the indications for CAS besides the commercially available applications. This study reports on CAS supported resection of recurrent carcinomas within the pelvis in three patients.

\section{Methods}

The CAS system Surgigate ${ }^{\circledR}$, Module "Spine" was used with navigated chissels from the PAO-Module (Medivision, Oberdorf, Switzerland) in all cases because no specific application are available to support the resection of tumors.

Patient 1. A 37 y old male with a recurrent chondrosarcoma located presacral on the left side of S 2 (Fig. 1). Because the recurrent tumor was small and located next to the sacral cortex a tumor resection including the anterior cortex of S2 preserving the nerval roots of $\mathrm{S} 2$ were performed. 
For registration four titanium K-wires placed into the iliac chrest in local anesthesia and the most convex part of the promontorium were used as landmarks.

The operation was done in supine position using a median distal laparotomy with a transperitoneal approach. The DRB was placed on the left iliac crest. The tumor was localized according to the MRI presacral on the left side in height of S 2 with the navigated pointer, the ventral cortex of the sacrum was osteotomized preserving the sacral channel with the navigated chisel (Fig. 2) and the tumor could be removed in one block.

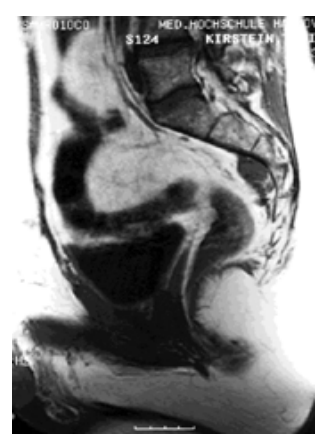

Fig. 1: The MRI showing the chondrosarcoma directly in front of the sacrum.

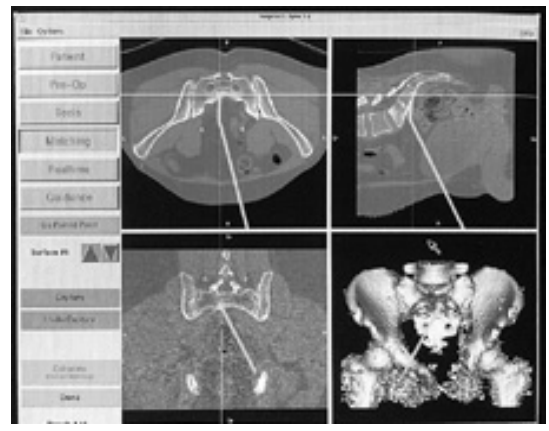

Fig. 2: Screenshot during the navigated resection of the chondrosarcoma.

Patient 2. A 52 y old female with a recurrent chordoma of the sacrum. CT scans showed an infiltration of the sacrum up to S2 bilateral and an infiltration of the left SI-joint (Fig. 3). The operation was done in prone position. After preparation of the soft tissues the DRB was attached to the sacral body, registration was done using anatomical landmarks for paired point registration: the processus spinosus of $\mathrm{S} 1$ and $\mathrm{S} 2$, the processus transversus bilateral of S1. Surface matching was done using the bony surface of the destructed sacrum. The operation was done using navigated chisels (Fig. 4). The sacrum was removed partially on the levels S1 and S2 including the nerval roots, furthermore the left SI-joint was removed.

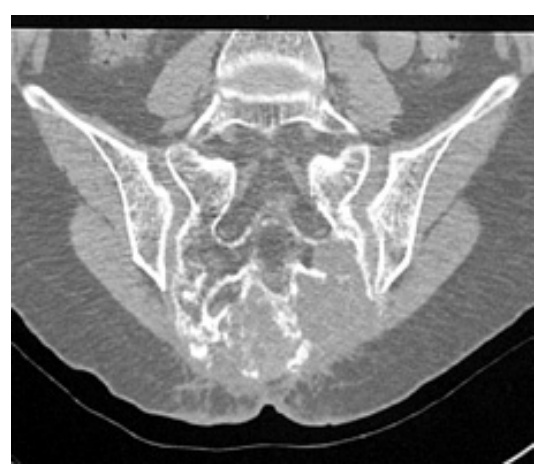

Fig. 3: A CT reformation of the recurrent chordoma showing the infiltration of the sacrum.

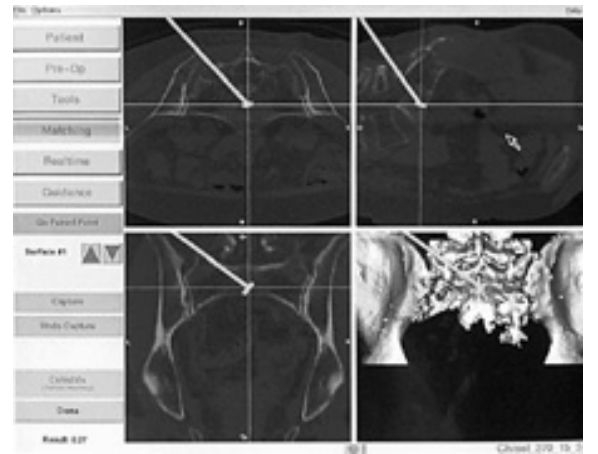

Fig. 4: The monitor view during the navigated procedure showing the navigated chissel (green) while resecting the tumor. 
Patient 3. A 39 y old female with a recurrent cervix carcinoma infiltrating the body S 1 on the left side (Fig. 5). An exenteration operation with a partial resection of the sacrum was done. Preoperatively 2 titanium pins (1.6 $\mathrm{mm}$ diameter) were inserted on the iliac crest bilaterally and a CT was obtained. The operation was done in 3 steps:

1. The patient in prone position doing a CAS supported lumbosacral stabilization to prevent an instability of the posterior pelvic ring due to the partial resection of the sacrum. CAS was used for the placement of the pedicle and the iliac screws. Additionally a hemilaminectomy of S 1 and S 2 and a posterior dissection of the sacroiliac ligaments was performed conventionally.

2. The patient was turned in supine position. The rectum and both ureters were mobilized. The tumor was infiltrating the left common iliac vein and the left obturator nerve, furthermore the left roots of L5 and S 1. The DRB was attached to the left iliac crest after dissection of the vessels and partial replacement with Gore-Tex was done. Paired point registration was done using the fiducials, the SIAS bilateral and the convexity of the promontorium, surface registration on both iliac bones. With navigated chisels an osteotomy of L5 and S 1 paramedian on the right side was performed (Fig. 6). The infiltrated S 1 was identified on the screen and the left S 2 body was resected as well. The tumor could be removed en bloc.

3. A corticospongous bone transplant of the left iliac crest was done for stabilization and the defect between L5 and the left ilium was covered. A Wolter plate was placed additionally for stabilization and the patient was mobilized with full weight bearing.

\section{Discussion and Conclusion}

Although existing navigation systems support specific steps as pedicle screw placement in the field of orthopaedic surgery, the provided tools allow an extension of indications.

Our experience is limited to three patients, yet, however we found the identification and the resection in critical regions like those close to the sacrum can be done with a high accuracy, if CAS supported. Further developments as CT- and MRI fusion or an intraoperative integration of imaging techniques like ultrasound could lead to improved results and a more extended spectrum of indications.

\section{References}

1. Langlotz U, Langlotz F, Hiogne D, et al. A Novel System for Complete THR Planning and Intraoperative Free-Hand Navigation. in CAOS Computer Assisted Orthopaedic Surgery. 2000. Davos, Switzerland.

2. Lavallee, S, P Sautot, J Troccaz, et al.: Computer-assisted spine surgery: A technique for accurate transpedicular screw fixation using CT data and a 3-D optical localizer. J Image Guid Surg, 1(1): 65-73,1995. 